ARTICLE

https://doi.org/10.1038/s41467-019-14106-0

OPEN

\title{
Clonal competition within complex evolutionary hierarchies shapes AML over time
}

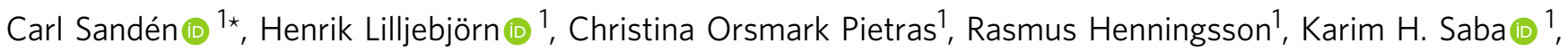
Niklas Landberg (10) 1,2, Hanna Thorsson', Sofia von Palffy (10) ', Pablo Peña-Martinez (1) 1, Carl Högberg1, Marianne Rissler ${ }^{1}$, David Gisselsson (10 1, Vladimir Lazarevic (10 ${ }^{2}$, Gunnar Juliusson², Helena Agerstam ${ }^{1}$ \& Thoas Fioretos $^{1 \star}$

Clonal heterogeneity and evolution has major implications for disease progression and relapse in acute myeloid leukemia (AML). To model clonal dynamics in vivo, we serially transplanted $23 \mathrm{AML}$ cases to immunodeficient mice and followed clonal composition for up to 15 months by whole-exome sequencing of 84 xenografts across two generations. We demonstrate vast changes in clonality that both progress and reverse over time, and define five patterns of clonal dynamics: Monoclonal, Stable, Loss, Expansion and Burst. We also show that subclonal expansion in vivo correlates with a more adverse prognosis. Furthermore, clonal expansion enabled detection of very rare clones with AML driver mutations that were undetectable by sequencing at diagnosis, demonstrating that the vast majority of $\mathrm{AML}$ cases harbor multiple clones already at diagnosis. Finally, the rise and fall of related clones enabled deconstruction of the complex evolutionary hierarchies of the clones that compete to shape AML over time.

\footnotetext{
${ }^{1}$ Division of Clinical Genetics, Department of Laboratory Medicine, Lund University, Lund, Sweden. ${ }^{2}$ Department of Hematology, Oncology and Radiation Physics, Skåne University Hospital, Lund, Sweden. *email: carl.sanden@med.lu.se; thoas.fioretos@med.lu.se
} 
ML arises through successive acquisition of genetic alterations, which creates complex hierarchies of clones with different combinations of mutations and chromosomal aberrations ${ }^{1,2}$. However, the extent of clonal heterogeneity at diagnosis and whether relapse clones are predominantly present at diagnosis or evolve during chemotherapy remain open questions $^{3-14}$. Patient-derived xenografts (PDXs) are the only model system that enables expansion of AML patient cells with preserved leukemia stem cell capacity ${ }^{15-18}$. Thus, PDXs have emerged as a critical model to study the response to chemotherapy and novel targeted therapies as well as potential resistance mechanisms, although the severe immunodeficiencies that allow human engraftment also make studies that rely on interactions between the leukemia and the immune system more challenging ${ }^{19,20}$. We hypothesized that serial transplantations over longer periods of time would expose inherent differences in proliferation and self-renewal capacity between competing clones and thus allow modeling of clonal dynamics in vivo. We also hypothesized that the emergence and disappearance of related clones would enable delineation of clonal hierarchies and detection of previously undetectable clones, thus unmasking clonal heterogeneity in AML.

\section{Results and discussion}

Patient-derived AML xenografts enable long-term modeling of clonal dynamics. We transplanted 26 AML cases to the NSG-S mouse model ${ }^{18}$ and allowed engraftment to proceed until the first signs of disease. This long-term approach generated high engraftment levels from the vast majority of samples $(23 / 26$; $88 \%$ ), encompassing a wide spectrum of recurrent AML aberrations (Fig. 1a-d, Table 1). All 26 patient samples and 84 xenografts were subjected to whole-exome sequencing (WES) to track clonal dynamics based on somatic mutations, copy number alterations (CNA) and copy-neutral losses of heterozygosity, making this the largest cohort of paired AML and xenograft samples to date $e^{9,21-23}$. In contrast to previous work, we also allowed clonal evolution to proceed until the development of disease in both primary and secondary recipients, yielding combined latencies of up to 15 months.

Cancer cell clones are defined as cell populations containing unique sets of genetic aberrations. Thus, we first identified clones based on all detected mutations using the PyClone algorithm ${ }^{24}$. With this approach, we found that the vast majority of cases (21/ 23 ; $91 \%)$ underwent changes in clonal composition from the patient samples to the derived xenografts, with 19 of 23 cases (83\%) exhibiting subclonal expansion in vivo and 13 of 23 cases (57\%) exhibiting subclonal reduction or loss (Supplementary Data 1-22). However, most of these clones were marked by presumed passenger or even synonymous mutations with limited or no functional significance. Hence, we thenceforth restricted the analysis to clones defined by recurrent AML mutations ${ }^{25}$ or CNA. For the subsequent analyses, cells with additional nonrecurrent presumed passenger mutations were thus not considered to constitute distinct subclones. With regard to clones with AMLassociated aberrations, we identified five patterns of clonal dynamics in AML PDXs herein referred to as: Monoclonal, Stable, Loss, Expansion and Burst (Fig. 1e).

AML xenografts undergo extensive clonal competition. The composition of clones with different AML-associated alterations was found to change significantly from the patient sample to the xenografts in as many as 17 of 23 cases (74\%), thereby enabling inference of clonal hierarchies and detection of rare clones. The remaining 6 of 23 AML cases (26\%) exhibited the same clonal composition at diagnosis and in the xenografts. In five of these cases, the patient sample and the PDXs contained the same single AML clone, referred to as Monoclonal, only differing in terms of presumed passenger mutations (Fig. 2a; additional cases in Supplementary Fig. 1). In one case, a minor subclone was maintained at a low frequency in vivo, referred to as Stable (Fig. 2b).

In 4 of 23 AML cases (17\%), the major clone at diagnosis was lost or reduced in the xenografts, giving way to a parental clone containing all but one or two of the AML mutations, referred to as Loss (Fig. 3a; Supplementary Fig. 2). The presence of these parental clones at diagnosis could not be predicted based on sequencing of the patient samples alone, as the mutations defining the lost subclones had allele frequencies as high as those pertaining to the parental clones. Thus, the Loss pattern unveiled clonal diversity at diagnosis and identified late events during leukemogenesis, including mutations in CDKN2A and RUNX1T1. Strikingly, four cases lost clones with NRAS or KRAS mutations (Fig. 4), mirroring a common clinical scenario where $R A S$ mutations are lost from diagnosis to relapse ${ }^{7}$. In multiple cases, the subclones did not start to decrease until the second generation of xenografts, suggesting that certain late mutations may allow or even promote initial expansion in vivo but eventually exhaust the leukemia stem cell population.

In 11 of 23 AML cases (48\%), the xenografts emanated from a minor subclone in the diagnostic sample, referred to as Expansion (Fig. 3b; Supplementary Fig. 3). The expanding clones had average allele frequencies of only $5 \%$ in the patient samples, and in three cases the clones were undetectable at diagnosis and only revealed by expansion in vivo (AML-34: Fig. 3b, AML-16: Supplementary Fig. 3a and AML-17: Supplementary Fig. 3b). In AML-34, clonal expansion revealed the presence of four separate subclones with losses of different segments and alleles of chromosome 7 , none of which were detected by WES $(81 \times)$ or routine clinical karyotyping at diagnosis. Interestingly, one xenograft carried neither the NRAS (p.G12V) mutation nor any of these losses of chromosome 7 but instead revealed the presence of another clone carrying a different NRAS mutation (p.G12S) and a different partial loss of chromosome 7 . These strikingly parallel evolutionary trajectories suggest that the emergence of specific mutations in cancer may be driven less by stochastics and more by the intrinsic and extrinsic evolutionary pressures on the individual leukemia. It also indicates that certain leukemias may harbor pools of distinct but genetically similar clones. Loss of chromosome 7 is a risk-defining alteration according to the ELN 2017 guidelines $^{26}$, and provided that this holds true also at low frequencies, detection of these subclones at diagnosis would have changed the risk assessment from intermediate to adverse. This suggests that AML patients may harbor unexpected clonal complexity with clinical implications, including rare clones with CNAs that are not detectable by sequencing at diagnosis. The expansion of minor subclones led to complete loss of parental clones and sibling clones carrying other recurrent AML mutations, thus unmasking clonal hierarchies. The losses of the BCOR mutations in AML-21 and AML-54 (Supplementary Fig. 3c and Fig. S3i), the WT1 mutation in AML-7 (Supplementary Fig. 4), the CEBPA and ETV6 mutations in AML-54 (Supplementary Fig. 3i) as well as the NRAS mutations in AML-17 and AML-34 (Supplementary Fig. 3b and Fig. 3b) demonstrate not only their late acquisition but also evolutionary branching with multiple clones containing mutually exclusive driver mutations, something that cannot be inferred from sequencing of diagnostic samples alone.

In 2 of $23 \mathrm{AML}$ cases (9\%), rare clones with allele frequencies as low as the background at diagnosis expanded in primary xenografts but were lost in secondary recipients, referred to as Burst (Fig. 3c; Supplementary Fig. 4). This is the first report to demonstrate that changes in clonal composition can be 

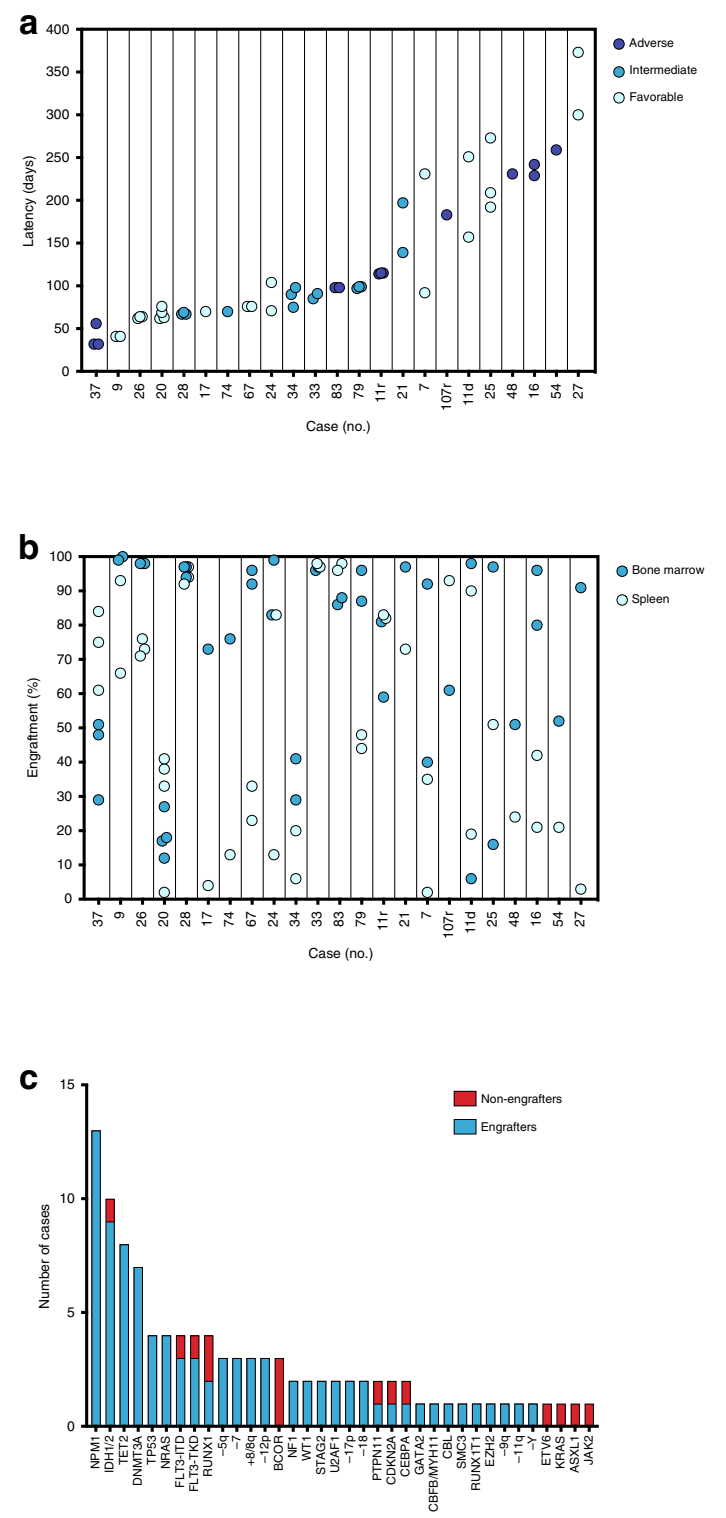
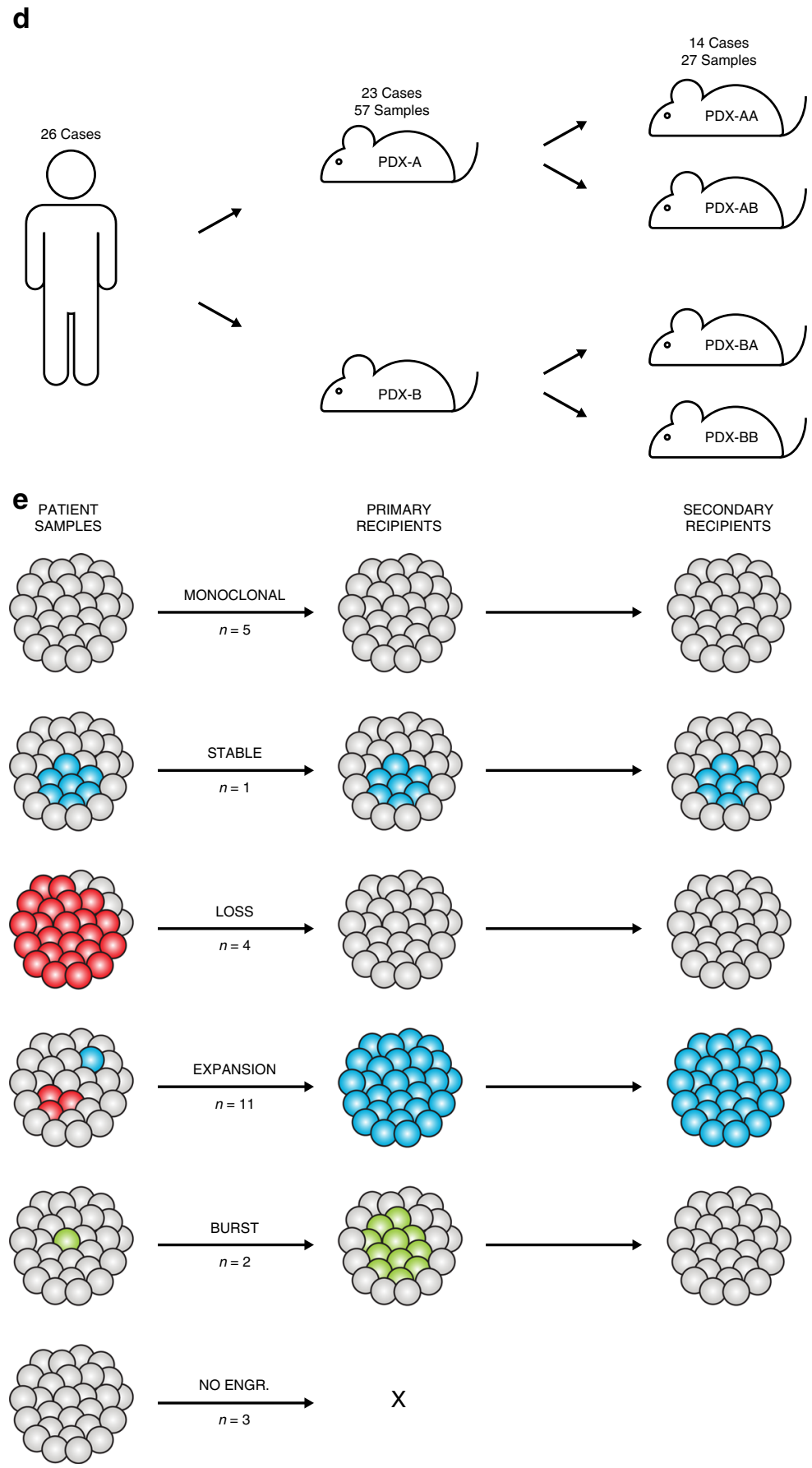

$X$

Fig. 1 Patient-derived AML xenografts undergo extensive clonal competition across multiple generations. Latency (a) and engraftment levels (b) in primary recipient mice, identically ordered by average disease latency. Risk categories were based on the ELN 2017 guidelines. c Engraftment of primary AML samples based on the presence of recurrent AML mutations. $\mathbf{d}$ Schematic overview of the generation and nomenclature of PDXs. e Patterns of clonal dynamics identified as leukemias were transplanted to primary and secondary recipient mice. $n$ denotes the number of cases that follow each pattern.

reversed after secondary transplantation, suggesting that AML cells undergo continuous clonal competition across multiple generations in vivo and that certain genetic aberrations may confer initial expansion at the expense of long-term self-renewal.

AML PDXs are genetically stable with moderate reproducibility. In many cases, the emergence of even very rare clones was notably consistent between replicate xenografts, as with the NRAS clone in AML-21, the -7 clones in AML-34 and the transient burst of the SMC3 clone in primary xenografts from AML-11r.
However, certain patterns were less consistent, such as the loss of the NRAS clone observed in two of five xenografts from AML-20, and the varying expansion of the NF1, FLT3 and PTPN11 clones in different xenografts from AML-7.

To further dissect the reproducibility, we performed limiting dilution analysis of two patient samples with substantial changes in clonal composition. We then analyzed the xenografts generated by the minimal required cell doses, which should be the most susceptible to random variation. In AML-21, all five xenografts displayed the same expansion of the minor NRAS clone seen in 
Table 1 Patient characteristics of transplanted AML cases.

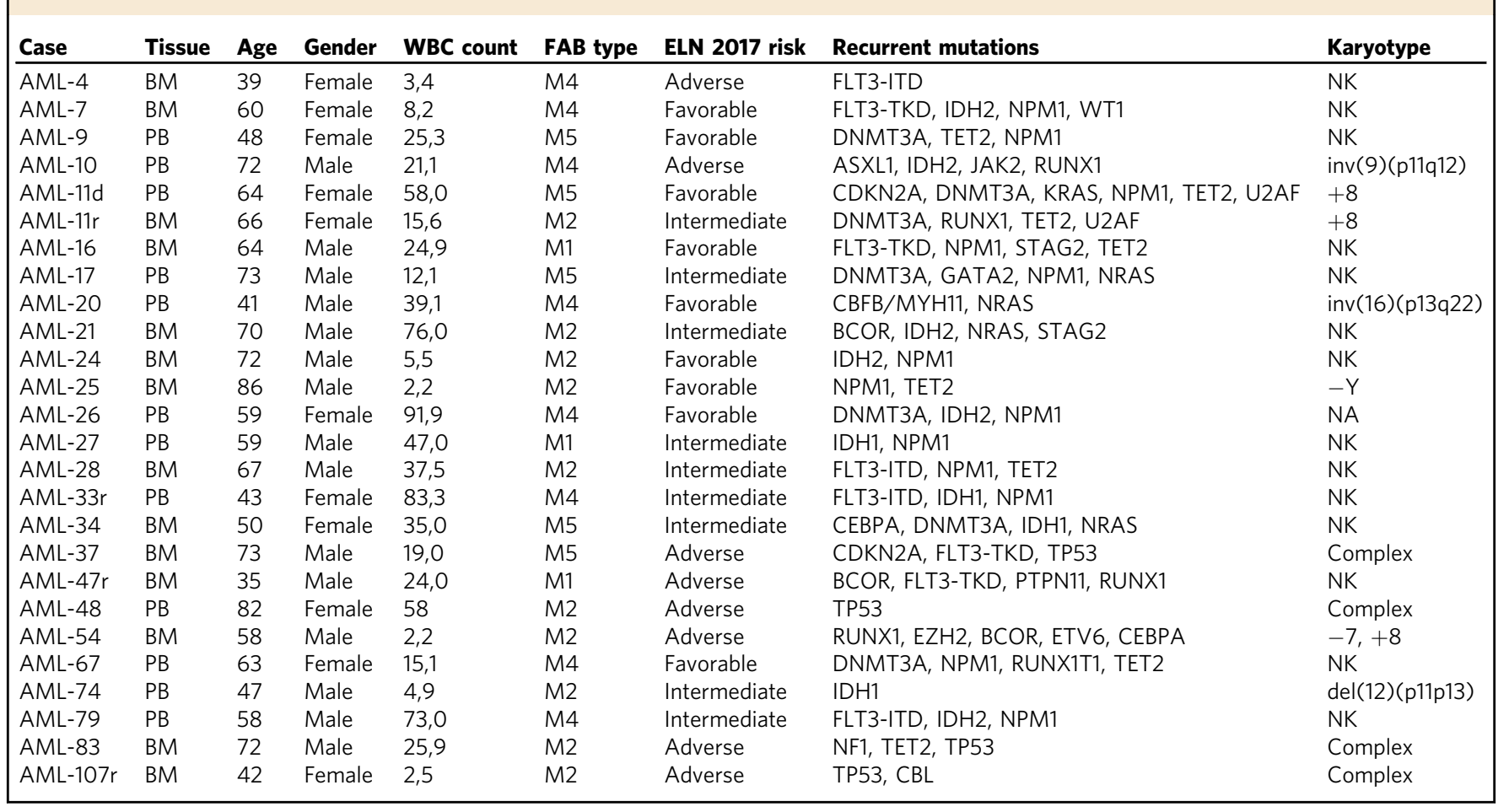

the other PDXs (Supplementary Fig. 3a). However, the three PDXs generated from AML-34 all displayed different clonal compositions (Fig. 3b).

Thus, engraftment seems to be largely dictated by clonal fitness but still subject to stochastic variation, especially at lower cell doses. However, this variability should decrease over time as clonal heterogeneity is progressively reduced and more clones are permanently lost. We would thus speculate that the most consistent clonal contribution is obtained with high cell numbers from serially passaged xenografts.

Despite the striking changes in clonal composition, AML PDXs were found to be remarkably genetically stable, with fewer acquired mutations than the commonly used retroviral MLL-AF9 mouse model ${ }^{27,28}$ and fewer novel CNAs than PDXs from other tissues $^{29}$. At least 15 of the 19 recurrent AML alterations that emerged in the xenografts could be retraced to the diagnostic samples, and WES only identified 0.44 passenger mutations per passage, with a majority of the xenografts not gaining any novel mutations despite an average latency of 110 days in vivo.

AML xenografts can reveal but not predict relapse clones. To determine if clonal evolution in vivo mirrors the development from diagnosis to relapse, we analyzed matched samples from seven AML cases (Supplementary Fig. 5). In two cases, the clonal dynamics in patients and xenografts converged. In AML-7, the xenografts revealed the presence of a subclone with an NF1 mutation that was otherwise undetectable at diagnosis and emerged as the major clone at relapse. In AML-11, the engrafting clones mirrored the loss of the CDKN2A, KRAS and NPM1 mutations seen at relapse but did not recapitulate the gain of a RUNX1 mutation and del(11)(q23) observed at relapse. However, in four cases the clonal dynamics diverged and the clones that emerged or persisted in vivo were lost at relapse. One case displayed no change in clonal composition. Thus, we show that AML PDXs can reveal rare relapse clones in diagnostic samples but in accordance with previous work ${ }^{21}$, we find that the clonal evolution in xenografts does not consistently mimic that from diagnosis to relapse. This may be due to differences in the human and mouse microenvironments or the lack of selective pressure from chemotherapy in the PDX model. In terms of engraftment characteristics, we did not see a difference between the 20 diagnostic samples and the three relapse samples transplanted in our cohort but observed that all three relapse samples contained multiple clones and exhibited expansion of minor subclones in vivo, possibly contributing to the increased aggressiveness at relapse.

Clonal dynamics in AML PDXs correlates with genetic risk. We also found a correlation between clonal dynamics and genetic risk. All six cases $(6 / 6 ; 100 \%)$ with adverse risk genetics were found to contain subclones at diagnosis that expanded in vivo, compared to four of the six cases $(4 / 6 ; 67 \%)$ with intermediate risk and only three of the 11 cases $(3 / 11 ; 27 \%)$ classified as favorable risk ( $p=0.008$; Fisher's exact test, two-sided) (Fig. 4). Thus, clonal and functional heterogeneity could contribute to the poor prognosis ${ }^{30,31}$ as well as the traditionally high engraftment capacity 22,32 of adverse and intermediate risk AML patients. Conceivably, leukemias with large repertoires of potentially expansive clones would more likely contain or be able to generate clones with the capacity to withstand chemotherapy and reemerge to cause relapse. The observed difference in genetic risk largely stems from differences in NPM1 mutation status, with nine of ten $N P M 1^{w t}$ cases (90\%) but only 4 of $13 N P M 1^{m u t}$ cases (31\%) displaying expansion of minor subclones in vivo. It is thus also possible that NPM1-mutated AML is less prone to clonal branching and/or expansion, a feature that could potentially contribute to the relatively favorable prognosis of this subtype. In contrast to genetic risk, we did not see a correlation between clonal dynamics and latency, indicating that the expansion rates of the respective clones are more impactful than their frequencies. 
a
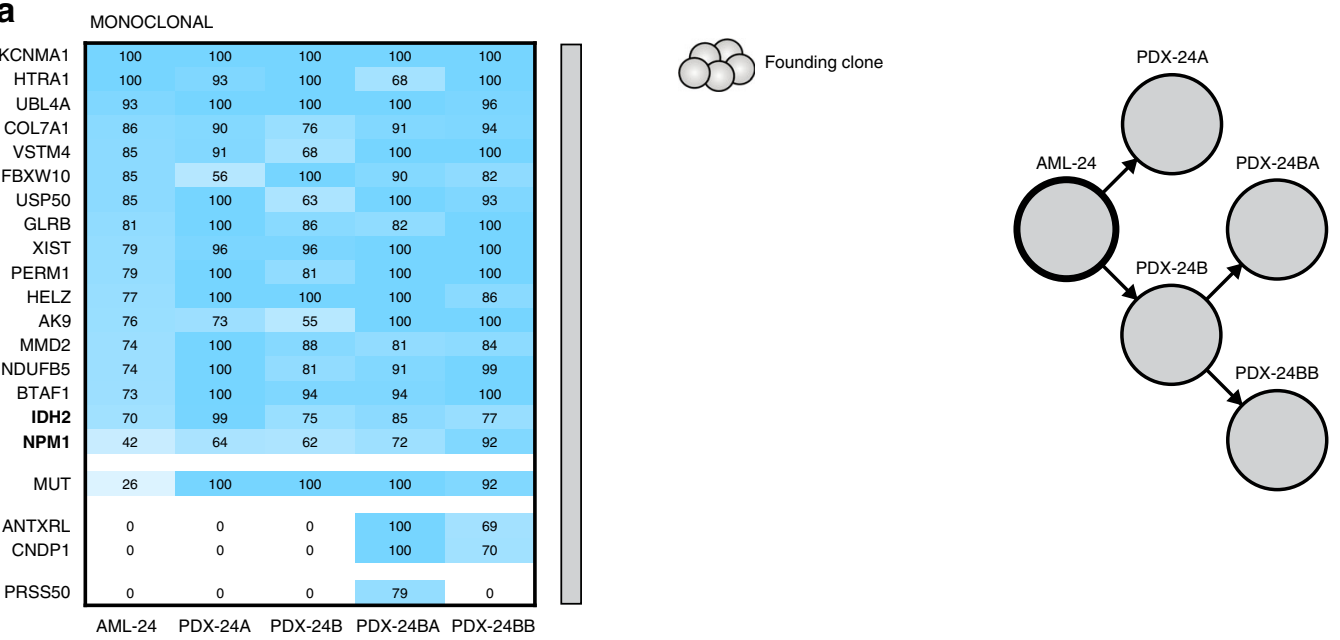

b

\begin{tabular}{|c|c|c|c|c|c|c|c|}
\hline \multirow[b]{2}{*}{ ASPM } & \multicolumn{7}{|c|}{ STABLE } \\
\hline & 100 & 95 & 99 & 100 & 100 & 92 & 95 \\
\hline PDS5B & 100 & 62 & 58 & 70 & 56 & 53 & 54 \\
\hline AMPD1 & 100 & 82 & 95 & 100 & 92 & 100 & 100 \\
\hline IQCG & 96 & 87 & 94 & 97 & 87 & 98 & 100 \\
\hline TAS2R5 & 94 & 100 & 92 & 93 & 100 & 92 & 100 \\
\hline ALG6 & 93 & 99 & 94 & 84 & 100 & 100 & 100 \\
\hline RLBP1 & 90 & 95 & 86 & 86 & 100 & 100 & 94 \\
\hline F7 & 87 & 100 & 100 & 100 & 100 & 123 & 100 \\
\hline TET2 & 85 & 100 & 100 & 100 & 98 & 95 & 100 \\
\hline METTL5 & 82 & 100 & 100 & 100 & 76 & 100 & 93 \\
\hline OCA2 & 79 & 93 & 78 & 98 & 73 & 70 & 99 \\
\hline ADAMTSL1 & 75 & 84 & 87 & 100 & 100 & 90 & 100 \\
\hline MAPRE2 & 74 & 85 & 96 & 80 & 89 & 100 & 100 \\
\hline FAM193A & 71 & 100 & 100 & 74 & 86 & 100 & 91 \\
\hline LOC440040 & 71 & 71 & 80 & 70 & 99 & 100 & 100 \\
\hline ABCG5 & 67 & 92 & 83 & 100 & 87 & 100 & 100 \\
\hline TET2 & 64 & 80 & 94 & 85 & 100 & 100 & 100 \\
\hline SLC10A2 & 61 & 100 & 100 & 100 & 100 & 100 & 100 \\
\hline FBXO31 & 58 & 81 & 100 & 100 & 52 & 100 & 82 \\
\hline C19ort24 & 54 & 100 & 100 & 100 & 82 & 60 & 97 \\
\hline NPM1 & 49 & 63 & 68 & 70 & 57 & 77 & 65 \\
\hline FLT3-ITD & + & + & + & + & + & + & + \\
\hline $\mathrm{LOH}(13)$ & 26 & 12 & 19 & 33 & 26 & 26 & 21 \\
\hline
\end{tabular}
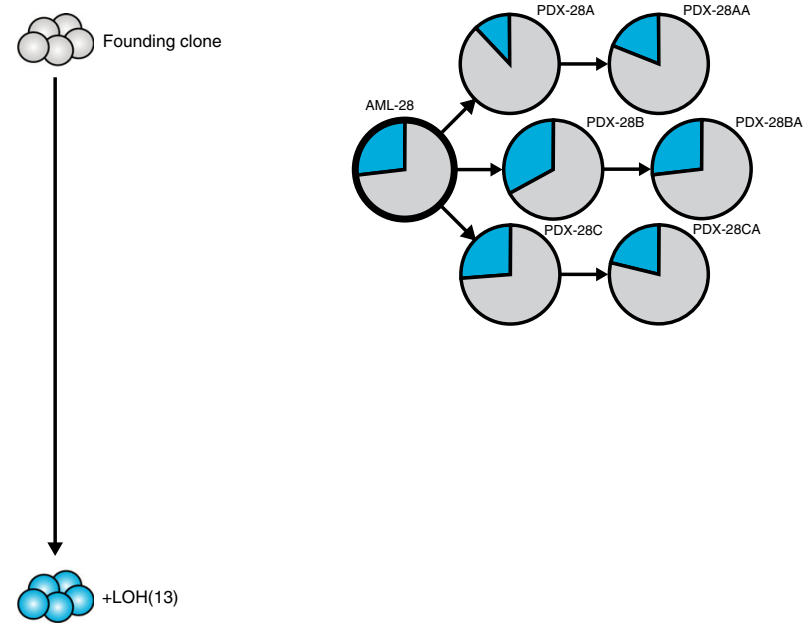

Fig. 2 The clonal composition is maintained in a minority of AML xenografts. a A representative AML case with the Monoclonal pattern of clonal dynamics, where the patient sample only contains one detectable clone with AML-associated mutations or CNAs and this clone constitutes the entire xenografts. In AML-24, a single clone with IDH2 and NPM1 mutations gave rise to both the two primary and the two secondary xenografts, differing only in terms of nonrecurrent presumed passenger mutations. Left, the percentage of cells in patient samples and corresponding xenografts estimated to carry each genetic aberration, based on variant allele frequencies of identified mutations and b-allele frequencies of copy number alterations and copy-neutral losses of heterozygosity. Colored bars indicate defining mutations for each clone. Clones are represented by the same color throughout each panel. Middle, inferred clonal hierarchy. Right, proportions of each clone at diagnosis (AML, thick circles) and in xenografts (PDX, thin circles). Clones were defined by the presence of one or more recurrent AML mutations, CNAs or losses of heterozygosity (indicated in bold). $\mathbf{b}$ The only AML case with the Stable pattern of clonal dynamics, where clones in the patient sample retain their relative proportions in the xenografts. In AML-28, a subclone with loss of heterozygosity of chromosome 13 maintained its frequency from the patient sample in all three primary and three secondary xenografts. The presence of FLT3-ITD is denoted by + , as the rearrangement in this case is detectable but not quantifiable by WES.

Clonal dynamics is validated by single-cell sequencing. Singlecell sequencing has emerged as a powerful tool to decipher cancer cell heterogeneity. Transcriptional analysis has identified and characterized different types of functionally distinct AML cells $^{33,34}$, and single-cell genotyping has unmasked clonal complexity ${ }^{35-37}$ with implications for both disease progression ${ }^{38}$ and treatment resistance ${ }^{39,40}$. However, single-cell DNA sequencing is dependent on amplification of small amounts of DNA, which generates both false-positives and false-negatives ${ }^{41}$, making it difficult to identify rare clones at diagnosis that are readily detectable upon expansion in xenografts.

We performed scRNA-seq with the $10 \times$ Genomics platform on one such case and identified monosomy 7 in the diagnostic sample and two xenografts from AML-34, where clones with losses of either allele emerged in different frequencies. This analysis confirmed the expansion of clones with monosomy 7 in the xenografts, with PDX-34B dominated by the -7 (II) clone and PDX-34C only containing the -7 (I) clone and clones with partial losses of chromosome 7 that were too small to detect by this method (Supplementary Fig. 6). As with WES, the clones with monosomy 7 were not reliably detectable by scRNA-seq at diagnosis, as the potentially positive cells were rare and of low confidence (frequency: $1.1 \%$; expected background rate: $0.2 \%$ ), and did not form a distinct cluster in the gene expression space. This analysis thus confirmed the clonal dynamics observed by WES and underscored the potential of PDXs to uncover otherwise undetectable clonal heterogeneity in cancer.

AML xenografts unmask clonal heterogeneity at diagnosis. By generating and sequencing xenografts, we could infer the 
a

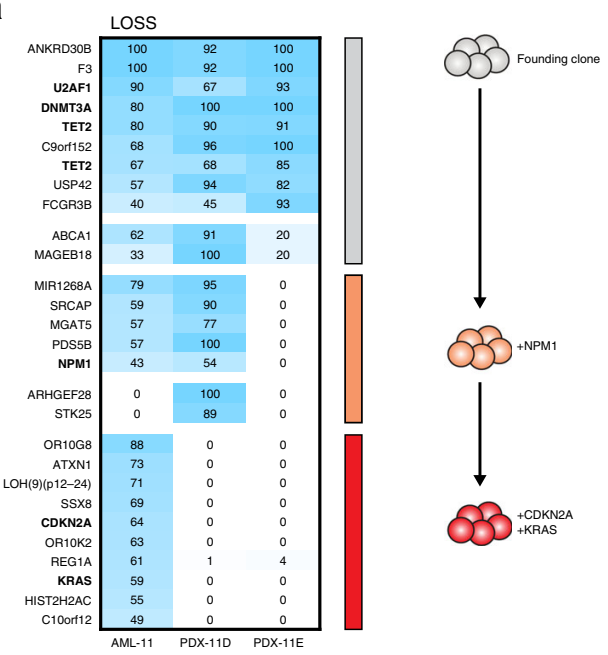

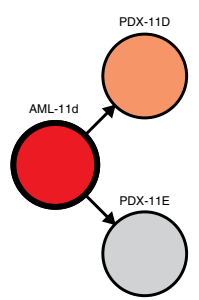

b

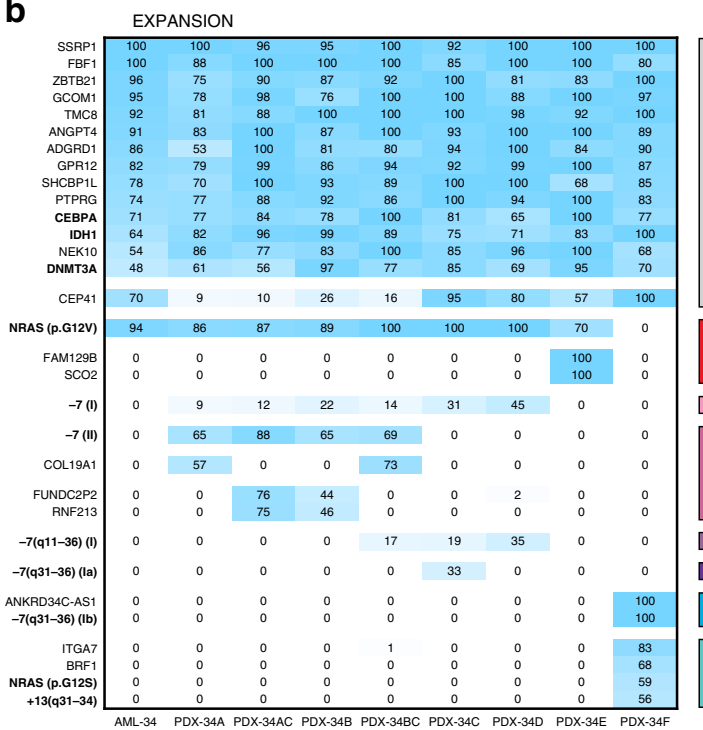

C

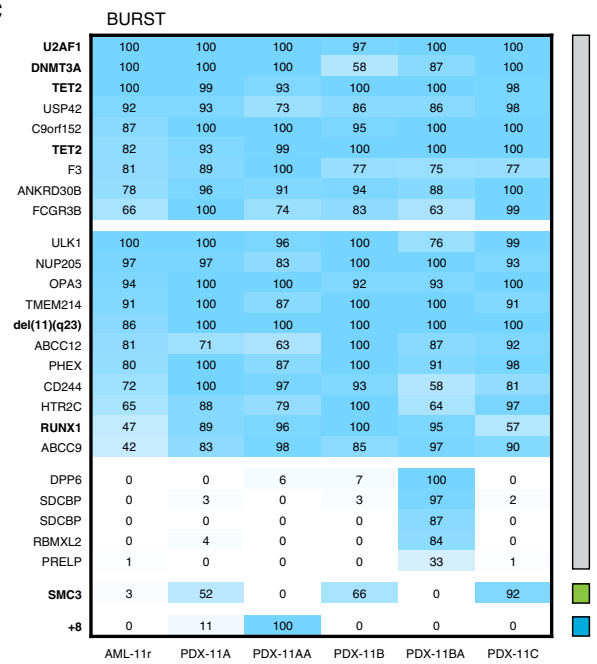

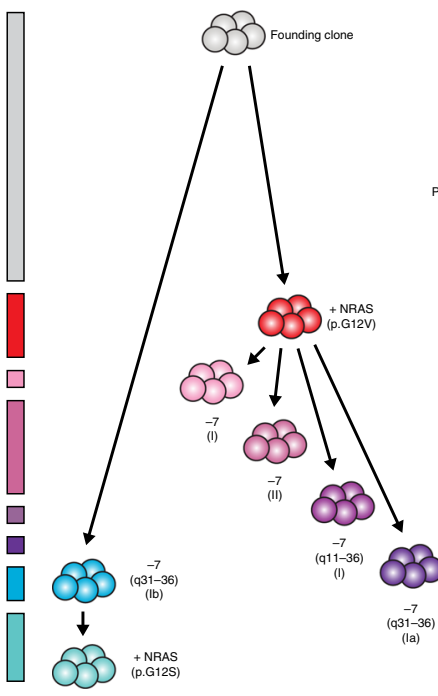
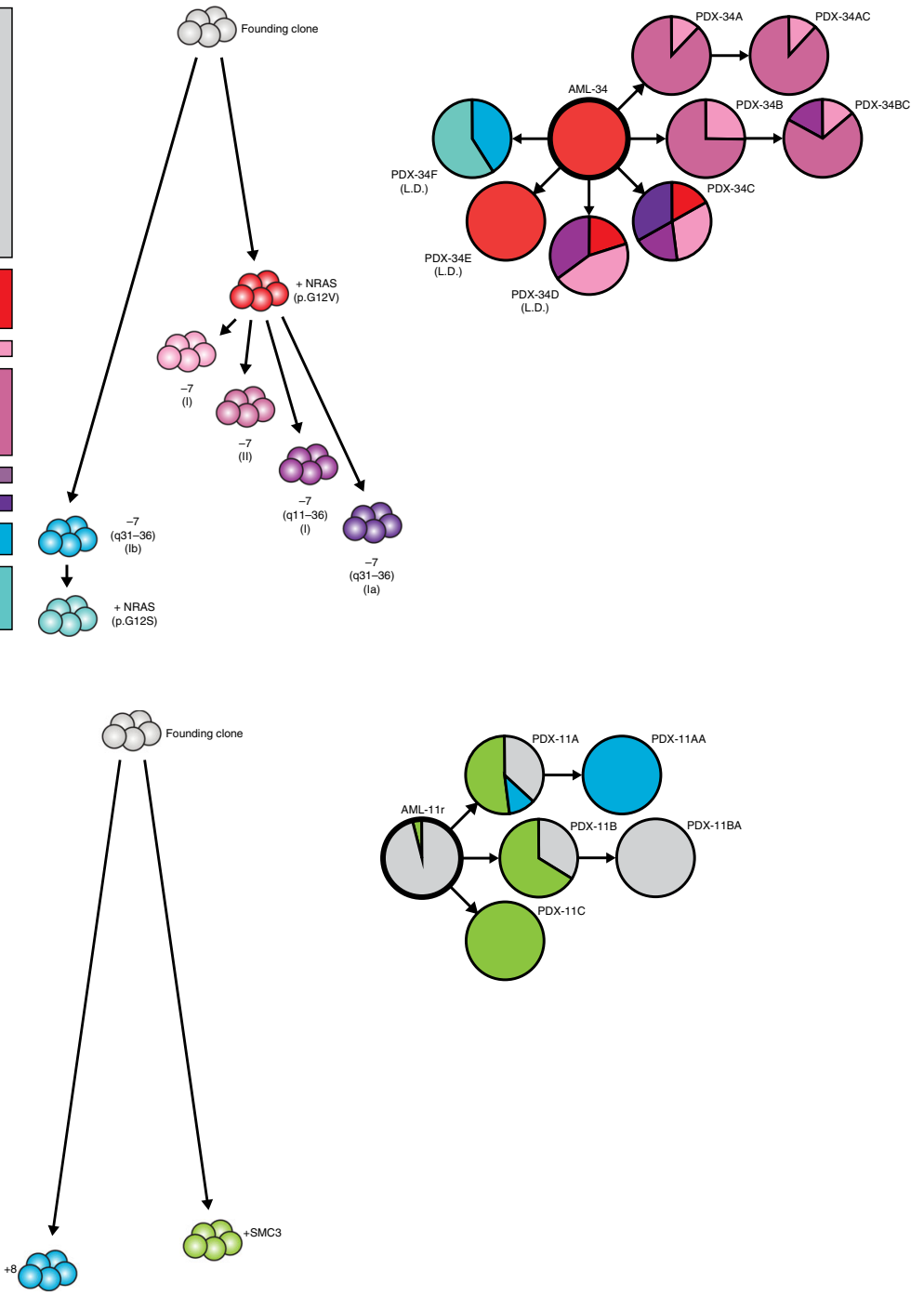

presence of multiple clones with distinct sets of recurrent AML mutations or CNAs in 18 of 23 AML cases (78\%) at diagnosis. This is higher than what was recently seen in a smaller cohort (4/ $11 ; 36 \%)^{22}$ of single primary recipient mice by a 19 -gene panel, and higher than what has been reported based on whole-genome

sequencing (WGS) of diagnostic samples without xenotransplantation $(27 / 50 ; 54 \%)^{42}$ and WES of matched diagnosis and relapse patient samples $(23 / 50 ; 46 \%)^{7}$. Strikingly, at least 11 of 23 cases $(48 \%)$ contained clones that were present at diagnosis but only revealed by xenografting, thereby also identifying five 
Fig. 3 The clonal composition changes in the majority of AML xenografts. a A representative AML case with the Loss pattern of clonal dynamics, where a subclone in the patient sample is reduced or completely lost in the xenografts. In AML-11d, the dominant clone in the patient sample, with CDKN2A and KRAS mutations, was lost in both xenografts, resulting in engraftment with one of two parental clones. Left, the percentage of cells in patient samples and corresponding xenografts estimated to carry each genetic aberration, based on variant allele frequencies of identified mutations and $b$-allele frequencies of copy number alterations and copy-neutral losses of heterozygosity. Colored bars indicate defining mutations for each clone. Clones are represented by the same color throughout each panel. Middle, inferred clonal hierarchy. Right, proportions of each clone at diagnosis and in PDXs. Clones were defined by the presence of one or more recurrent AML mutations, CNAs or losses of heterozygosity (indicated in bold). L.D. denotes samples transplanted at minimal cell dose based on limiting dilution analysis. b A representative AML case with the Expansion pattern of clonal dynamics, where a subclone in the patient sample expands to constitute the entirety of the xenografts. In AML-34, clones with one of two different NRAS mutations and five different partial or complete losses of chromosome 7, which were all undetectable at diagnosis, expanded to generate the xenografts. The heterozygous CEP41 mutation is present in the founding clone but located on chromosome 7 and thus lost in the -7 (II) clone but retained in the -7 (I) clone. I and II denote the two alleles of chromosome 7, whereas a and b represent distinct genetic events. c A representative AML case with the Burst pattern of clonal dynamics, where a subclone in the patient sample expands in primary xenografts but is lost in secondary xenografts. In AML-11r, a small subclone with an SMC3 mutation transiently expanded to make up the majority of both primary recipients but was lost in the two secondary recipients. The +8 aberration was detected at very low frequency in the diagnostic sample by routine clinical karyotyping.

recurrent AML genetic alterations (NF1, PTPN11, SMC3, TET2 $\mathrm{LoH}$ and -7$)$ that were not detected in the clinical diagnostic samples (Fig. 4). The presence of these clones at diagnosis could be inferred from detection in multiple independent lines of xenografts (e.g. AML-34; Fig. 3b), an approach that cannot be employed in studies comparing diagnostic and relapse samples. By applying this principle, we also identified rare diagnostic clones with CNAs, which are inherently difficult to detect by retrospective deep sequencing. Previous work has demonstrated the expansion of minor subclones in cases known to have multiple detectable clones ${ }^{21,23}$. However, the frequent emergence of undetectable subclones in seemingly monoclonal patients has not previously been shown and suggests that AML harbors more clonal heterogeneity at diagnosis than is detectable by standard methods. In the 11 cases with unmasked clones, the changes in clonal composition also revealed information about clonal hierarchies not discernable at diagnosis, including genetic aberrations shown to be acquired late during leukemogenesis, branching events and mutations occurring in separate subclones (e.g. AML21: Supplementary Fig. 3b and AML-7: Supplementary Fig. 4). Thus, our sequencing of PDXs shows AML patients to harbor more clonal heterogeneity than previously described. The presence of rare previously undetectable clones at diagnosis also raises the possibility that genetic changes from diagnosis to relapse to a larger extent than previously believed may result from competition between rare pre-existing diagnostic clones rather than clonal evolution throughout chemotherapy and remission.

In this study, we demonstrate that AML generally harbors multiple clones already at diagnosis, including rare clones that are not detectable at diagnosis but only revealed upon serial transplantation. We show that individual clones are genetically stable but undergo extensive and reversible clonal competition over time. We thus conclude that AML harbors a high degree of clonal and functional heterogeneity, with implications for disease biology and future therapeutic strategies in AML.

\footnotetext{
Methods

Patient-derived xenografts. Aspects of the study involving patient cells were approved by the Swedish Ethical Review Authority. Informed consent was obtained and all work was conducted in compliance with all relevant ethical regulations for work with human participants. Aspects of the study involving research animals were approved by the regional Animal Ethics Committee of Malmö/Lund and complied with all relevant ethical regulations for animal testing and research. Bone marrow and peripheral blood samples from AML patients were collected at the Department of Clinical Genetics, Skåne University Hospital after written informed consent. Mononuclear cells were prepared by lymphoprep separation (GE Healthcare) and viably frozen. For primary and secondary transplantations, cells were thawed and $\mathrm{T}$ cells depleted by either CD3 microbead separation (Miltenyi Biotec) or treatment with the OKT3 anti-CD3 antibody (BioXCell). Except where otherwise noted, a total of $\geq 5$ million cells were transplanted by tail vein injection
}

to sublethally irradiated NOD.Cg-Prkdc ${ }^{\text {scid }}$ Il2 2 rg $^{\text {tm1Wjl }}$ /SzJ-SGM3 (NSG-S) mice (250 cGy), a substrain of the NSG mouse overexpressing hGM-CSF, hIL-3 and hSCF (Jackson Laboratory). Mice were euthanized upon signs of serious illness or 1 year after transplantation, whereupon leukemic engraftment, defined as the percentage of $\mathrm{hCD} 45^{+} \mathrm{CD} 33^{+} \mathrm{CD} 3^{-} \mathrm{CD} 19^{-}$cells, was assessed by flow cytometry on an LSR Fortessa (BD Biosciences). For limiting dilution experiments, $10^{3}, 10^{4}, 10^{5}$ and $10^{6}$ cells were similarly transplanted and sacrificed after 16 weeks. For sequencing, leukemia cells from $\mathrm{PDX}$, defined as $\mathrm{hCD} 45^{+} \mathrm{CD} 33^{+} \mathrm{CD} 3{ }^{-} \mathrm{CD} 19^{-}$, were sorted by flow cytometry on a FACS Aria Fusion (BD Biosciences). The gating strategy is detailed in Supplementary Fig. 7.

Whole-exome sequencing. Somatic mutations, CNAs and losses of heterozygosity were detected by WES. DNA was isolated from patient bone marrow or peripheral blood and from sorted $\mathrm{CD} 45^{+} \mathrm{CD} 33^{+}$xenograft cells by the DNeasy Blood \& Tissue Kit (Qiagen). WES libraries were produced using the Nextera rapid capture exome kit (Illumina) or the human core exome kit (Twist Bioscience) and sequenced by paired end $2 \times 150$ bp sequencing on a NextSeq 500 (Illumina). After WES, adapter sequences were trimmed from the reads using cutadapt 1.9.1. To minimize the impact of sequence reads of murine origin, the reads were aligned to both human reference genome hg19 and murine reference genome mm10 using bwa mem ${ }^{43}$. Reads of murine and ambiguous origin were then removed using disambiguate ${ }^{44}$. After alignment and murine read removal, the median coverage of targeted regions was $113 \times$. Somatic mutations were detected using strelka 2.8.4 and freebayes $1.1 .0^{46}$ followed by a custom filter for identifying somatic variants. A freebayes somatic score was calculated as the sum of the log10-scaled genotype likelihoods (GL) for the normal sample to differ from the alternative genotype and the tumor sample to differ from the normal genotype $\left(\mathrm{GL}_{\mathrm{NS}, \mathrm{NGT}}-\mathrm{GL}_{\mathrm{NS}, \mathrm{TGT}}+\right.$ $\mathrm{GL}_{\mathrm{TS}, \mathrm{TGT}}-\mathrm{GL}_{\mathrm{TS}, \mathrm{NGT}}$, where $\mathrm{NS}=$ normal sample, $\mathrm{TS}=$ tumor sample, $\mathrm{NGT}=$ normal genotype, and TGT $=$ tumor genotype). Variants with a strelka somatic snv score (QSS) above 140, strelka somatic variant score (QSI) above 100, or freebayes somatic score above 90 were considered somatic variants. Variants suspected to originate from murine reads not removed using the read filter strategy were identified and removed by comparing against a mouse variant blacklist ${ }^{47}$ created by aligning whole genome sequencing reads from the Mouse Genome Project ${ }^{48}$ to human genome hg19. All positions with remaining somatic variants were regenotyped in all samples from this patient using freebayes 1.1.0 to determine the frequency in all relevant samples. Complete lists of somatic variants and their frequencies in each sample are presented as Supplementary Data 23-44. Variant allele frequencies of recurrently mutated genes were validated in patient samples using a Nextera rapid capture custom kit targeting 109 genes recurrently mutated in AML that was sequenced to a median coverage of $587 \times$ (Supplementary Data 23 $-44)$. Copy number aberrations and loss of heterozygozity variants were detected from whole-exome data using cnvkit $0.9 .1^{49}$. Germline variants fulfilling the following criteria were used to calculate SNV b-allele ratio for loss of heterozygosity detection: (1) freebayes quality (QUAL) > 300); (2) combined coverage within samples from one patient $>200$ and (3) only two detectable genotypes with allele frequency between 0.4 and 0.6 in the germline sample. Complete lists of CNAs in each sample are presented as Supplementary Data 45-58.

Single-cell sequencing. For single-cell sequencing, $\mathrm{CD} 45^{+} \mathrm{CD} 33^{+}$cells from patient and xenograft samples were sorted as described above. Single-cell 3' RNA-seq libraries were prepared using the Chromium Single-cell 3' reagent kit v3 (10x genomics). Single cells were separated into gel beads-in-emulsion (GEMs) using the Chromium controller (10× genomics) and full-length transcripts containing cellular and unique molecular barcodes were produced inside individual GEMs, after which the GEMs were broken and the barcoded transcripts amplified using PCR. The amplified full-length cDNA was fragmented enzymatically, the fragment ends were repaired and A-tailed, and Illumina-compatible adaptor oligos were ligated onto the 


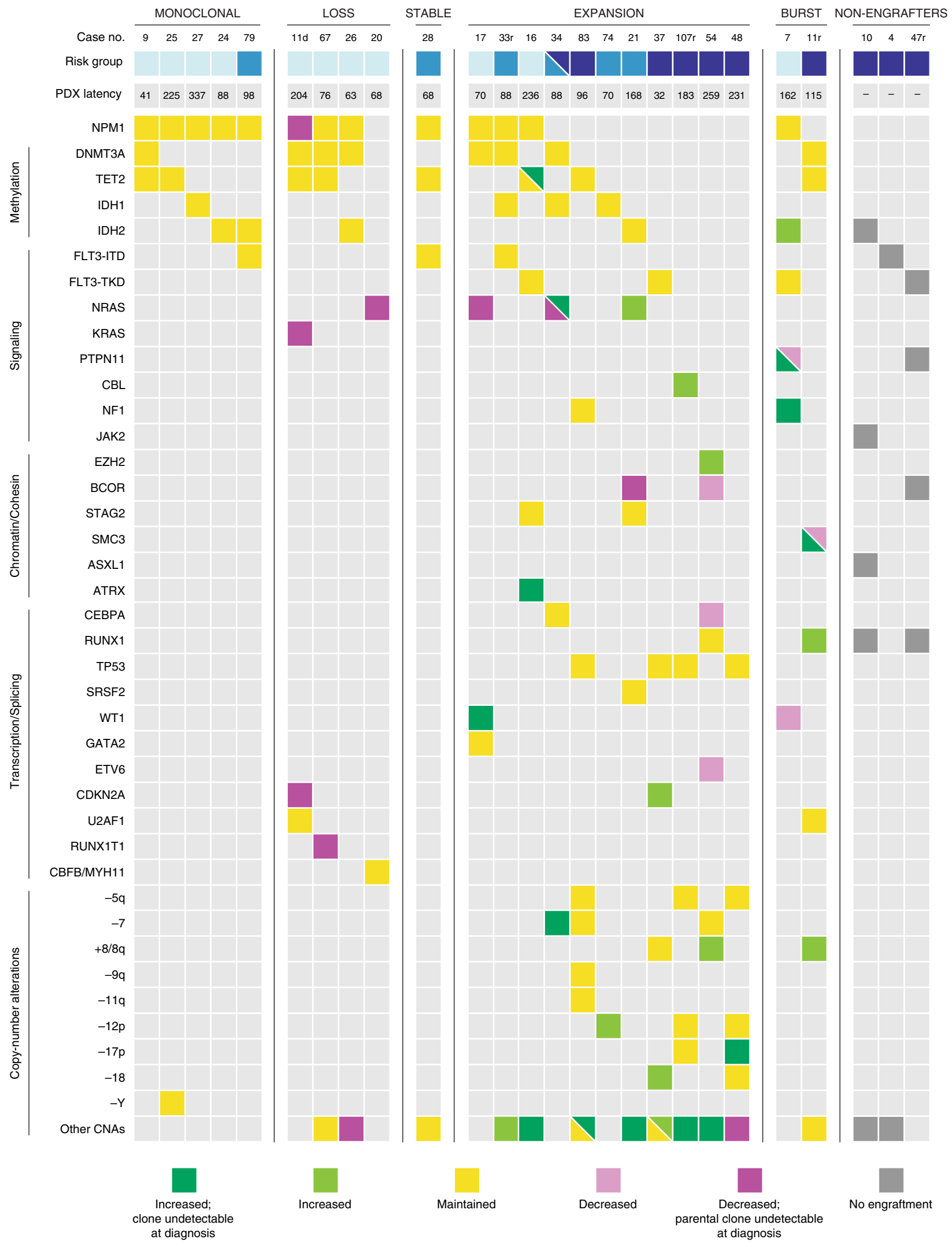

fragments. The fragments where then amplified and extended to include full-length sequencing adaptors by PCR. The final libraries were sequenced on a Nextseq 500 (Illumina) with 34,000-36,000 reads/cell. Single-cell gene expression data were produced from the sequencing data using the cell ranger pipeline $(10 \times$ genomics). Genotyping and detection of loss of chromosome 7 in the single cells was performed by genotyping 54 single nucleotide polymorphisms (SNPs) with coverage in the

single-cell data and known assignment to either the maternal or paternal chromosome 7 as determined by exome sequencing in samples with loss of chromosome 7 . The SNPs were genotyped in each cell using vartrix ${ }^{50}$, the genotype data were pooled across SNPs and a single read containing a genotype assigned to either chromosome 7 was interpreted as presence of that chromosome 7 in that cell. A minimum of six reads from a single chromosome 7 without any reads from the other chromosome 7 
Fig. 4 Clonal competition in AML xenografts unmasks rare clones and correlates with genetic risk. Recurrent genetic alternations of all AML cases and changes in allele frequencies from patient samples to xenografts. Dark green: mutation with increased frequency that was undetectable at diagnosis. Light green: mutation with increased frequency. Yellow: mutation with maintained frequency. Light purple: mutation with reduced frequency. Dark purple: mutation with reduced frequency, revealing a parental clone that was undetectable at diagnosis. Gray: mutation in nonengrafting sample. CNA copy number alteration. Relapse samples are denoted by " $r$ " after the case number. Risk category classification based on the ELN 2017 guidelines. Light blue: favorable. Medium blue: intermediate. Dark blue: adverse. The dual coloring for AML-34 reflects the classification as intermediate based on information from the diagnostic sample alone and the classification as adverse when incorporating information derived from xenografts.

was interpreted as loss of chromosome 7. Gene expression data and chromosome 7 genotyping data were visualized using Seurat $\mathrm{v}^{51}$.

Clonal analysis. Analysis of clonal composition based on both presumed driver and passenger mutations was performed using the PyClone algorithm ${ }^{24}$. The analysis of clones defined by recurrent AML aberrations was performed manually and validated by PyClone. First, the percentage of cells carrying each genetic aberration was calculated by adjusting the variant allele frequency for ploidy, based on the copy number analysis. Percentages were capped at $100 \%$. The clonal hierarchy was then determined as the solution to the clonal structure that required the least diversion from the observed allele frequencies. In cases with ambiguous clonal hierarchies, the presence of potential subclones was determined by comparing the variant allele frequencies of the potential subclone and the potential parental clone using the Mann -Whitney $U$ nonparametric statistical test with a threshold of $p<$ 0.05 . For AML-34, the identification of multiple clones with losses of different segments of the two alleles of chromosome 7 was performed by quantification of the heterozygous somatic mutation in CEP41 as well as SNVs on chromosome 7 that differed between the two alleles and thus displayed different frequencies in xenografts dominated by different clones.

\section{Data availability}

The datasets generated during the current study fall under the GDPR regulations for sharing of personal data and will therefore be made available in the EGA-SE depository upon its completion. Until then, the datasets are available from the corresponding authors upon request through the following DOIs: https://doi.org/10.17044/NBIS/G000015 (WES dataset) and 10.17044/NBIS/G000016 (scRNA-seq dataset).

Received: 27 March 2019; Accepted: 17 December 2019; Published online: 05 February 2020

\section{References}

1. Bullinger, L., Dohner, K. \& Dohner, H. Genomics of acute myeloid leukemia diagnosis and pathways. J. Clin. Oncol. 35, 934-946 (2017).

2. Ferrando, A. A. \& Lopez-Otin, C. Clonal evolution in leukemia. Nat. Med. 23, 1135-1145 (2017).

3. Christopher, M. J. et al. Immune escape of relapsed AML cells after allogeneic transplantation. N. Engl. J. Med. 379, 2330-2341 (2018).

4. Corces-Zimmerman, M. R., Hong, W. J., Weissman, I. L., Medeiros, B. C. \& Majeti, R. Preleukemic mutations in human acute myeloid leukemia affect epigenetic regulators and persist in remission. Proc. Natl Acad. Sci. USA 111, 2548-2553 (2014).

5. Ding, L. et al. Clonal evolution in relapsed acute myeloid leukaemia revealed by whole-genome sequencing. Nature 481, 506-510 (2012).

6. Garg, M. et al. Profiling of somatic mutations in acute myeloid leukemia with FLT3-ITD at diagnosis and relapse. Blood 126, 2491-2501 (2015).

7. Greif, P. A. et al. Evolution of cytogenetically normal acute myeloid leukemia during therapy and relapse: an exome sequencing study of 50 patients. Clin. Cancer Res. 24, 1716-1726 (2018).

8. Griffith, M. et al. Optimizing cancer genome sequencing and analysis. Cell Syst. 1, 210-223 (2015).

9. Hirsch, P. et al. Genetic hierarchy and temporal variegation in the clonal history of acute myeloid leukaemia. Nat. Commun. 7, 12475 (2016).

10. Kronke, J. et al. Clonal evolution in relapsed NPM1-mutated acute myeloid leukemia. Blood 122, 100-108 (2013).

11. Parkin, B. et al. Clonal evolution and devolution after chemotherapy in adult acute myelogenous leukemia. Blood 121, 369-377 (2013).

12. Shlush, L. I. et al. Identification of pre-leukaemic haematopoietic stem cells in acute leukaemia. Nature 506, 328-333 (2014).

13. Sood, R. et al. Somatic mutational landscape of AML with inv(16) or t $(8 ; 21)$ identifies patterns of clonal evolution in relapse leukemia. Leukemia $\mathbf{3 0}$, 501-504 (2016).

14. Uy, G. L. et al. Dynamic changes in the clonal structure of MDS and AML in response to epigenetic therapy. Leukemia 31, 872-881 (2017).
15. Krevvata, M. et al. Cytokines increase engraftment of human acute myeloid leukemia cells in immunocompromised mice but not engraftment of human myelodysplastic syndrome cells. Haematologica 103, 959-971 (2018).

16. Lapidot, T. et al. A cell initiating human acute myeloid leukaemia after transplantation into SCID mice. Nature 367, 645-648 (1994).

17. Sanchez, P. V. et al. A robust xenotransplantation model for acute myeloid leukemia. Leukemia 23, 2109-2117 (2009).

18. Wunderlich, M. et al. AML xenograft efficiency is significantly improved in NOD/SCID-IL2RG mice constitutively expressing human SCF, GM-CSF and IL-3. Leukemia 24, 1785-1788 (2010).

19. Goyama, S., Wunderlich, M. \& Mulloy, J. C. Xenograft models for normal and malignant stem cells. Blood 125, 2630-2640 (2015).

20. Townsend, E. C. et al. The public repository of xenografts enables discovery and randomized phase II-like trials in mice. Cancer Cell 30, 183 (2016).

21. Klco, J. M. et al. Functional heterogeneity of genetically defined subclones in acute myeloid leukemia. Cancer Cell 25, 379-392 (2014).

22. Paczulla, A. M. et al. Long-term observation reveals high-frequency engraftment of human acute myeloid leukemia in immunodeficient mice. Haematologica 102, 854-864 (2017).

23. Wang, K. et al. Patient-derived xenotransplants can recapitulate the genetic driver landscape of acute leukemias. Leukemia 31, 151-158 (2017).

24. Roth, A. et al. PyClone: statistical inference of clonal population structure in cancer. Nat. Methods 11, 396-398 (2014).

25. Papaemmanuil, E. et al. Genomic classification and prognosis in acute myeloid leukemia. N. Engl. J. Med. 374, 2209-2221 (2016).

26. Dohner, H. et al. Diagnosis and management of AML in adults: 2017 ELN recommendations from an international expert panel. Blood 129, 424-447 (2017).

27. Hyrenius-Wittsten, A. et al. De novo activating mutations drive clonal evolution and enhance clonal fitness in KMT2A-rearranged leukemia. Nat. Commun. 9, 1770 (2018).

28. Kotani, S. et al. Molecular pathogenesis of disease progression in MLLrearranged AML. Leukemia https://doi.org/10.1038/s41375-018-0253-3 (2018).

29. Ben-David, U. et al. Patient-derived xenografts undergo mouse-specific tumor evolution. Nat. Genet. 49, 1567-1575 (2017)

30. Medeiros, B. C., Othus, M., Fang, M., Appelbaum, F. R. \& Erba, H. P. Cytogenetic heterogeneity negatively impacts outcomes in patients with acute myeloid leukemia. Haematologica 100, 331-335 (2015).

31. Bochtler, T. et al. Clonal heterogeneity as detected by metaphase karyotyping is an indicator of poor prognosis in acute myeloid leukemia. J. Clin. Oncol. 31, 3898-3905 (2013).

32. Pearce, D. J. et al. AML engraftment in the NOD/SCID assay reflects the outcome of AML: implications for our understanding of the heterogeneity of AML. Blood 107, 1166-1173 (2006).

33. Petti, A. A. et al. A general approach for detecting expressed mutations in AML cells using single cell RNA-sequencing. Nat. Commun. 10, 3660 (2019).

34. van Galen, P. et al. Single-cell RNA-Seq reveals AML hierarchies relevant to disease progression and immunity. Cell 176, 1265-1281.e1224 (2019).

35. Hughes, A. E. et al. Clonal architecture of secondary acute myeloid leukemia defined by single-cell sequencing. PLoS Genet. 10, e1004462 (2014).

36. Paguirigan, A. L. et al. Single-cell genotyping demonstrates complex clonal diversity in acute myeloid leukemia. Sci. Transl. Med. 7, 281 re282 (2015).

37. Potter, N. et al. Single cell analysis of clonal architecture in acute myeloid leukaemia. Leukemia 33, 1113-1123 (2019).

38. Pellegrino, M. et al. High-throughput single-cell DNA sequencing of acute myeloid leukemia tumors with droplet microfluidics. Genome Res. 28, 1345-1352 (2018).

39. McMahon, C. M. et al. Clonal selection with RAS pathway activation mediates secondary clinical resistance to selective FLT3 inhibition in acute myeloid leukemia. Cancer Discov. 9, 1050-1063 (2019).

40. Smith, C. C. et al. Heterogeneous resistance to quizartinib in acute myeloid leukemia revealed by single-cell analysis. Blood 130, 48-58 (2017).

41. Nangalia, J., Mitchell, E. \& Green, A. R. Clonal approaches to understanding the impact of mutations on hematologic disease development. Blood 133, 1436-1445 (2019) 
42. Cancer Genome Atlas Research Network. Genomic and epigenomic landscapes of adult de novo acute myeloid leukemia. N. Engl. J. Med. 368, 2059-2074 (2013).

43. Li, H. Aligning sequence reads, clone sequences and assembly contigs with BWA-MEM. Preprint at https://arxiv.org/abs/1303.3997 (2013).

44. Ahdesmaki, M. J., Gray, S. R., Johnson, J. H. \& Lai, Z. Disambiguate: an opensource application for disambiguating two species in next generation sequencing data from grafted samples. F1000Res. 5, 2741 (2016).

45. Kim, S. et al. Strelka2: fast and accurate calling of germline and somatic variants. Nat. Methods 15, 591-594 (2018).

46. Garrison, E. \& Marth, G. Haplotype-based variant detection from short-read sequencing. Preprint at https://arxiv.org/abs/1207.3907 (2012).

47. Salm, M., Schelhorn, S.-E., Lancashire, L. \& Grombacher, T. pdxBlacklist: Identifying artefactual variants in patient-derived xenograft samples. bioRxiv https://doi.org/10.1101/180752 (2017).

48. Keane, T. M. et al. Mouse genomic variation and its effect on phenotypes and gene regulation. Nature 477, 289-294 (2011)

49. Talevich, E., Shain, A. H., Botton, T. \& Bastian, B. C. CNVkit: genome-wide copy number detection and visualization from targeted DNA sequencing. PLoS Comput Biol. 12, e1004873 (2016).

50. Vartrix. https://github.com/10XGenomics/vartrix (2018).

51. Stuart, T. et al. Comprehensive integration of single-cell data. Cell 177, 1888-1902 e1821 (2019).

\section{Acknowledgements}

This work was supported by the Swedish Childhood Cancer Fund, the Swedish Cancer Society, the Swedish Research Council, the Medical Faculty at Lund University, the Royal Physiographic Society in Lund, the Knut and Alice Wallenberg Foundation, and the ISREC Foundation by a joint grant to the Swiss Cancer Center, Lausanne, CREATE Health Cancer Center, and the Medical Faculty at Lund University from the Biltema Foundation. We also thank the Center for Translational Genomics at Lund University and Clinical Genomics Lund, SciLifeLab, for providing single-cell sequencing service. Open access funding provided by Lund University.

\section{Author contributions}

C.S., H.L., C.O.P., H.Å. and T.F. designed the study. C.S., K.H.S., H.T., S.V.P., P.P.-M., C.H. and M.R. performed the experiments. H.L., C.O.P., N.L., V.L. and G.J. collected patient material and clinical data. C.S., H.L., C.O.P., R.H., K.H.S., H.T., D.G., H.Å. and T.F analyzed the data. C.S. and T.F. wrote the manuscript. All authors read and approved the final version of the manuscript.

\section{Competing interests}

The authors declare no competing interests.

\section{Additional information}

Supplementary information is available for this paper at https://doi.org/10.1038/s41467019-14106-0.

Correspondence and requests for materials should be addressed to C.S. or T.F.

Peer review information Nature Communications thanks Lars Bullinger and the other, anonymous, reviewer(s) for their contribution to the peer review of this work.

Reprints and permission information is available at http://www.nature.com/reprints

Publisher's note Springer Nature remains neutral with regard to jurisdictional claims in published maps and institutional affiliations.

cc (i) Open Access This article is licensed under a Creative Commons Attribution 4.0 International License, which permits use, sharing, adaptation, distribution and reproduction in any medium or format, as long as you give appropriate credit to the original author(s) and the source, provide a link to the Creative Commons license, and indicate if changes were made. The images or other third party material in this article are included in the article's Creative Commons license, unless indicated otherwise in a credit line to the material. If material is not included in the article's Creative Commons license and your intended use is not permitted by statutory regulation or exceeds the permitted use, you will need to obtain permission directly from the copyright holder. To view a copy of this license, visit http://creativecommons.org/ licenses/by/4.0/.

(C) The Author(s) 2020 